\title{
El desperdicio de alimentos: una perspectiva desde los estudiantes de Administración de Empresas de la UPS Guayaquil
}

\section{The waste of food: a perspective from the students of Business Administration of UPS Guayaquil}

\author{
Ercilia Franco Cedeño* \\ efranco@ups.edu.ec
}

\begin{abstract}
Resumen
Para disminuir el hambre en el mundo hoy se debate sobre la prioridad de producir más o reducir el desperdicio de alimentos. Ecuador también forma parte del debate porque teniendo una gran producción agrícola aún presenta niveles de desaprovechamiento de recursos y un gran sector de la población con niveles de pobreza que no les permite abastecerse de los nutrientes mínimos. Reducir el desperdicio debe ser prioridad de todos los estados y de todas las personas para remediar parte del impacto negativo al medio ambiente y además permitir que esos recursos sean aprovechados y se disminuya el número de personas con hambre.

El objetivo del estudio consistió en determinar las causas principales por las cuales las personas desperdician alimentos, y se hizo a través de una investigación de tipo cuantitativo, el que permitió establecer el lugar, horario, tipo de comida y la estrategia a elegir para reducir el desperdicio.
\end{abstract}

Los resultados determinaron que las personas no conocen el impacto ambiental negativo que se genera al desperdiciar alimentos.

\section{Palabras clave}

Impacto ambiental, desperdicio de alimentos.

\footnotetext{
* Ecuatoriana. Magister en Sistemas Integrados de Gestión de la Calidad,Ambiente y Seguridad. Universidad
} Politécnica Salesiana- Guayaquil. Docente Titular Auxiliar de la Carrera Administración de Empresas. 


\begin{abstract}
To reduce hunger in the world today debates is about the priority of producing more or to reduce food waste. Ecuador is also part of the debate because having a large agricultural production still has levels of waste of resources and a large sector of the population with poverty levels that do not allow them procure the minimum nutrients. Reduce waste should be a priority for all states and for all people to remedy some of the negative impact on the environment and also allow these resources be exploited and the number of hungry people can be decreased.
\end{abstract}

The aim of this study was to determine the main reasons why people waste food, and stepped through a quantitative analysis, which allowed us to establish the place, time, type of food and the strategy to choose to reduce waste.

The results determined that people do not know the negative environmental impact generated by wasting food.

\title{
Keywords
}

Environmental impact, food waste.

Forma sugerida de citar: Franco Cedeño, Ercilia (2016). El desperdicio de alimentos: una perspectiva desde los estudiantes de Administración de Empresas de la UPS Guayaquil. Revista Retos, 11(1), pp.51-64 .

\section{Introducción}

La población ecuatoriana tiene la ventaja de tener acceso a productos agrícolas a precios accesibles, especialmente en lo relativo a las frutas y verduras de temporada, es probable que la abundancia de recursos naturales no permita al ecuatoriano reflexionar sobre el descuido que tiene en ciertos hábitos alimenticios lo que ha generado la cultura del despilfarro, la cual tiende a generalizarse por la constante publicidad de las marcas comerciales de alimentos y es así que las diferentes fuentes de comunicación en cierto modo incentivan el uso desmedido de recursos naturales lo cual genera un grave impacto al medio ambiente (Calixto, 2015).

Se define como desperdicio a los alimentos inicialmente destinados al consumo y que son desechados o utilizados de forma alternativa, no alimentaria, ya sea por elección o porque se haya permitido que se estropeen o caduquen por negligencia (FAO, 2014a).

Las pérdidas por desperdicios de alimentos se deben principalmente a causas relacionadas con el comportamiento, como los hábitos de compra, preparación y consumo de alimentos, así como a la planificación del tiempo y la coordinación, y se ven influidas 
por técnicas de comercialización que animan a los consumidores a comprar más de lo necesario (HLPE, 2014).

Es cierto también que el mercado condiciona a las personas a adoptar costumbres en todos los aspectos por esto hay que tener presente que la cultura de las personas es resultado del sistema de producción vigente en cada sociedad (Escobar, 2012). Además los ecuatorianos aumentan el consumo de comidas fuera de casa especialmente por el ritmo de vida de las personas que ahora es más acelerado especialmente en las grandes ciudades.

A pesar de los esfuerzos del gobierno para prevenir, mitigar y remediar la contaminación ambiental en el Ecuador, especialmente agregando en la Constitución de la República del Ecuador entre otros el artículo No. 71, que da derechos a la naturaleza (Asamblea Nacional, 2008); también indicando explícitamente en el Objetivo No. 7 del Plan Nacional para el Buen Vivir 2013-2017, que garantizan los derechos de la naturaleza y se promueve la sostenibilidad ambiental (Senplades, 2013), se han aplicado controles y campañas para el cuidado ambiental por parte del Ministerio del Ambiente, pero aún estas campañas, sólo se han realizado a nivel de las empresas y se han difundido campañas televisivas de ahorro pero sólo para la energía eléctrica, por lo cual otro factor que influye en el desperdicio de alimen- tos es la falta de información y la falta de generación de las condiciones necesarias para que las personas aprovechen los residuos orgánicos de los hogares pues la mayoría de Municipios en el Ecuador no disponen de un sistema de recolección diferenciada de residuos, además tampoco existen medios accesibles o gestores que permitan sacar provecho a los residuos orgánicos de los hogares sin costo o que signifique algún beneficio para las familias.

Es importante conocer los factores que contribuyen en el desperdicio de alimentos pues impacta al medio ambiente ya que se amplía la frontera agrícola, disminuyendo extensos territorios de bosque y selva que dejan de actuar como sumidero de gases de efecto invernadero, o por otro lado el incremento en la crianza de ganado, genera grandes cantidades de metano, como también produce metano en su descomposición en los rellenos sanitarios (Xavier \& Gascón, 2014).

Representa una pérdida de recursos económicos para el consumidor, que paga por un bien que finalmente no consume, pierde recursos porque deja de recibir valores por desechar los alimentos, además, el dueño del restaurante también pierde, pues muchas veces desecha alimentos por los cuales canceló valores a su proveedor, y finalmente los Municipios podrían aprovechar esos residuos para la generación de energía. 
En Ecuador algunas personas aún desconocen que desperdiciar alimentos perjudica al medio ambiente, y sólo tratan de no caer en el desperdicio por ser solidarios con otras personas que no tienen recursos, por eso no se le da mucha importancia al tema.

Para comprender cabalmente el impacto medioambiental del desperdicio de comida, hay que considerar los recursos destinados al crecimiento, cosecha, procesamiento, transporte e incluso la cocción de nuestros alimentos (Abate, 2013, p. 40).

Las pérdidas y el desperdicio de alimentos (PDA) hacen referencia a una disminución de la masa de alimentos destinados originalmente al consumo humano, independientemente de la causa y en todas las fases de la cadena alimentaria, desde la cosecha hasta el consumo (CSA, 2014).

El 6\% de pérdidas de alimentos se da en América Latina y el Caribe mientras que un $7.9 \%$ de la población sufre de hambre y cada año la región pierde o desperdicia el $15 \%$ de sus alimentos disponibles, además el 28\% de desperdicio de alimentos se da en el consumo, $28 \%$ en producción, $22 \%$ en manejo y almacenamiento, $17 \%$ en mercado y distribución y $6 \%$ durante su procesamiento (FAO, 2014a).

Si Ecuador lograra disminuir sus desperdicios también lograría cumplir sus metas del milenio de reducir en número de personas las condiciones de hambre (FAO, 2014b).

Los hogares destinan el 24.40\% de sus ingresos al consumo de alimentos y bebidas no alcohólicas, y el decil ${ }^{1}$ 1 considerado el de más bajos ingresos y el de más altos ingresos decil 10, hay diferencias de consumos entre ellos, pues los de menores ingresos gastan el más alto porcentaje en pan y cereales con el $26 \%$ y en el de más altos ingresos gastan en carnes frescas y congeladas un $19 \%$. A nivel general los 220 productos de mayor consumo son el pan corriente, arroz blanco, colas y/o gaseosas, lecha entera en funda, pechuga, alas y piernas de pollo, pollo entero, queso de mesa, aceite de palma africana, huevos de incubadora, agua sin gas purificada, entre otros, concentran el 52\% del gasto en alimentos y bebidas no alcohólicas de los hogares (INEC, 2011-2012).

Ante un escenario donde un grupo de la población tiene dificultad de acceso a los alimentos especialmente por ingresos económicos, limitaciones en las cosechas y las fuentes de recursos naturales por efectos del cambio climático surge la necesidad de buscar respuesta a la siguiente pregunta de investigación: ¿Conocen los jóvenes el impacto ambiental negativo que produce el desperdicio de alimentos? Analizando desde la academia las causas por las cuales las personas desper-

1 Los deciles dividen un conjunto de observaciones en 10 partes iguales. 
dician alimentos, específicamente la comida preparada y a partir de estos resultados se puedan establecer estrategias que se apliquen en las aulas de clases para que los alumnos puedan reflexionar y con cambios fáciles de implementar en su comportamiento, se pueda reducir el desperdicio de alimentos lo cual contribuirá en mejorar la economía familiar, fomentar actitudes solidarias y hábitos que cuiden el medio ambiente.

\section{Metodología}

Para conocer las causas del desperdicio por parte de los jóvenes se aplicó en Mayo de 2015 un cuestionario que fue validado por expertos conocedores del tema y los datos fueron procesados en el programa estadístico informático SPSS.

La muestra corresponde a 335 estudiantes de los nueve niveles, de los tres horarios de la carrera de Administración de Empresas de la ciudad de Guayaquil. Se decidió realizar la investigación que primero contó con estudiantes porque una vez obtenidos los resultados se puede difundir fácilmente entre ellos estrategias de mejora y ellos serán replicadores de las buenas prácticas en sus hogares.

La encuesta consta de diez preguntas en las cuales se identificó el sexo del encuestado, edad, nivel que está cursando y se les consultó: ¿Cuál es el motivo por el cual se deja comida en el plato? ¿En qué lugar deja más co- mida? ¿En cuál de las tres comidas deja más desperdicios? ¿Si ha recibido alguna instrucción para no dejar alimentos en el plato? ¿Quién les dio alguna instrucción? ¿Si recibió sugerencias de estrategias para ahorrar alimentos? y ¿Si conocen del impacto ambiental del desperdicio de alimentos?

\section{Cálculo de la muestra}

$\mathrm{Al}$ momento de tomar la muestra en el mes de mayo de 2015, la Carrera de Administración de Empresas había matriculado a 2399 estudiantes, la cual fue la población de estudio y se contó con la fórmula para el cálculo de la población finita, donde se obtuvo el siguiente resultado:

$$
\begin{gathered}
n=\frac{Z^{2} P Q N}{e^{2}(N-1)+Z^{2} P Q} \\
\mathrm{n}=\frac{(1.96)^{2} \times(0.50)(0.50)(2399)}{(0.05)^{2}(2399-1)+(1.96)^{2}(0.50)(0.50)} \\
\mathrm{n}=332
\end{gathered}
$$

Finalmente se encuestó a 335 estudiantes de los nueve semestres y de las tres jornadas que la Carrera oferta, siendo el $66 \%$ de ellos mujeres y un $34 \%$ hombres.

Respecto a la edad el 80\% de los encuestados se encontraban entre los 18 y 23 años mientras que el 3\% tenía 17 años y para el $17 \%$ restante de ellos, su edad fue superior a los 23 años. 


\section{Resultados}

\section{Gráfico 1}

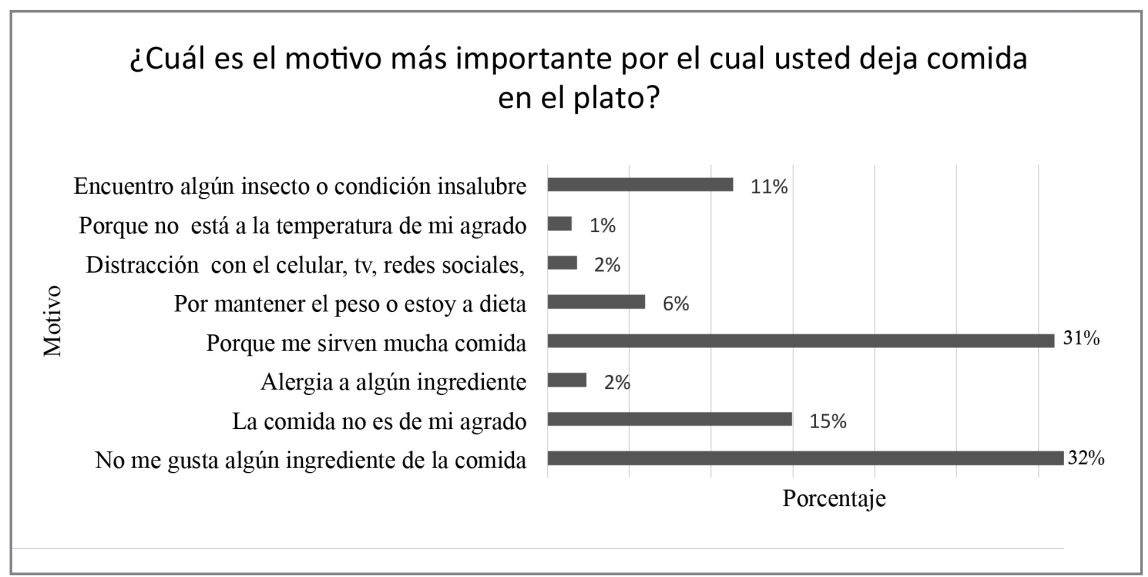

Fuente: Elaboración propia

El Gráfico 1 mostró que del total gún ingrediente de la comida, seguido de alumnos encuestados el $32 \%$ indicó con un 31\% que indicó como motivo, que dejó comida porque no le gustó al- porque le sirvieron mucha comida.

\section{Gráfico 2}

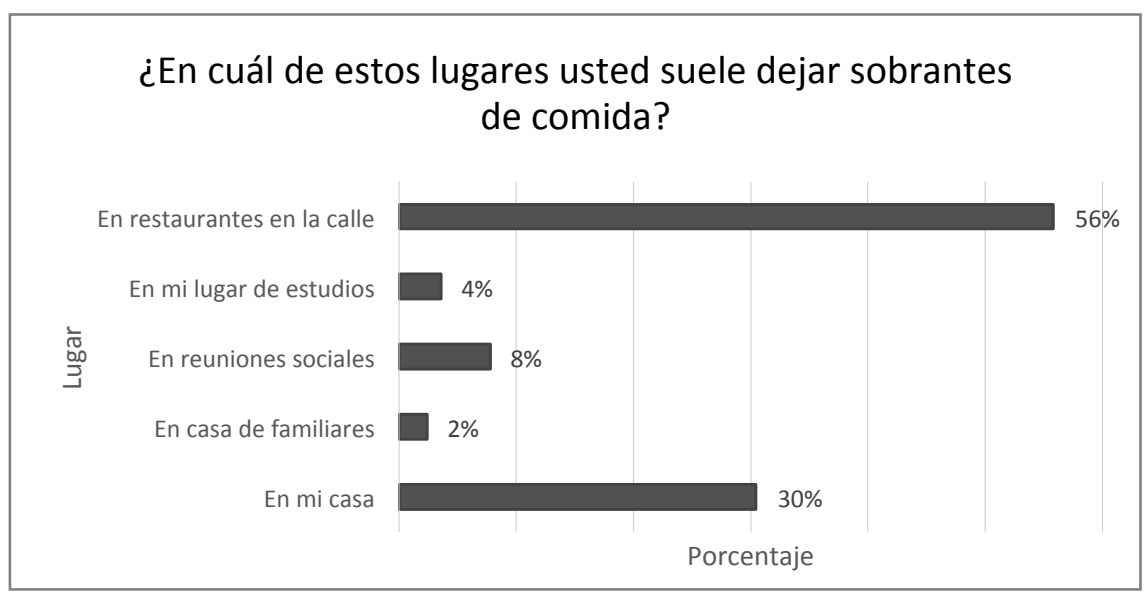

Fuente: Elaboración propia 
Según los resultados de la encuesta del Gráfico 2, el 56\% de los estudiantes indicó que dejó comida en restaurantes en la calle, luego con el $30 \%$ en su casa y en tercer lugar con el $8 \%$ en reuniones sociales.

\section{Gráfico 3}

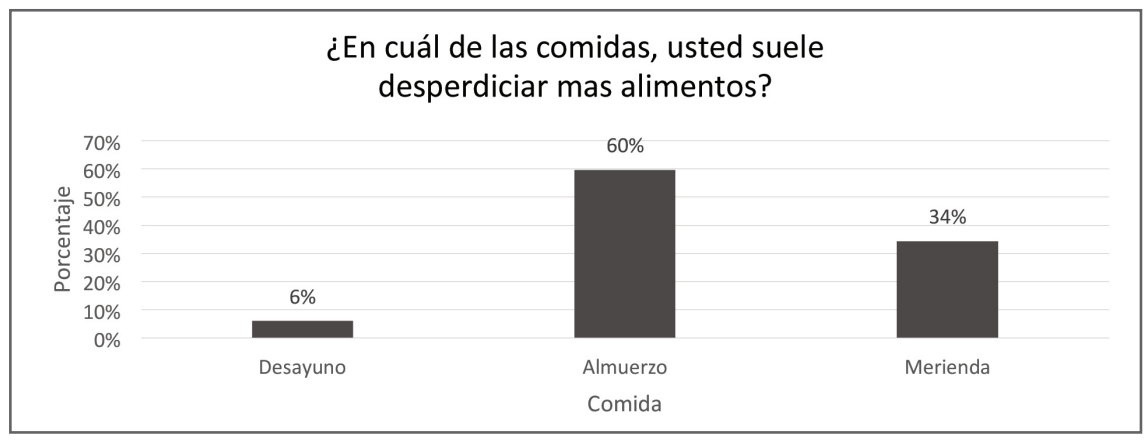

Fuente: Elaboración propia

El Gráfico 3 muestra que el 60\% de los encuestados dejó más comida en el almuerzo, un 34\% indicó que deja más comida en la merienda y sólo un 6\% manifestó que dejó comida en el desayuno.

\section{Gráfico 4}

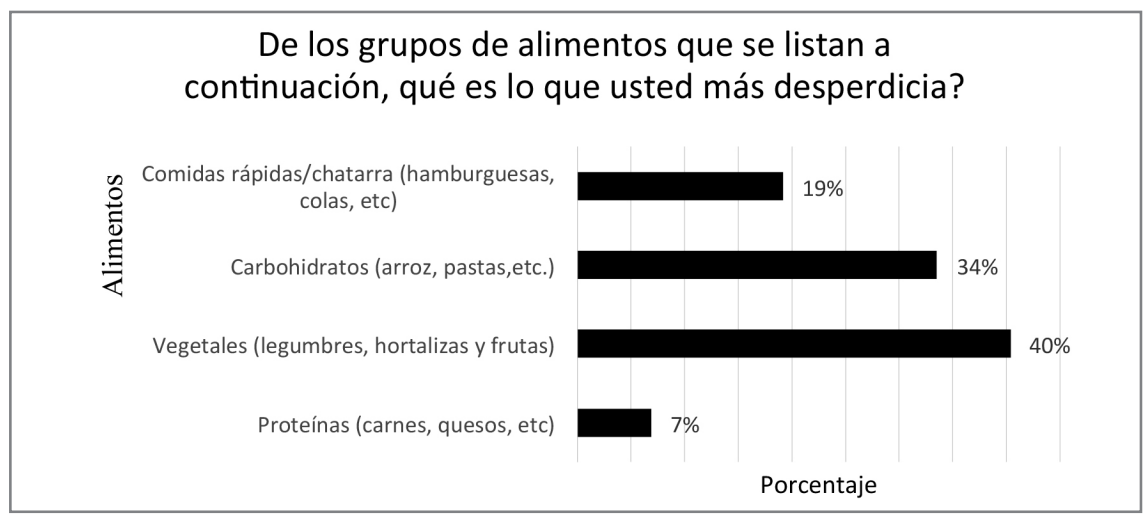

Fuente: Elaboración propia

En el Gráfico 4, el 40\% de los jóvenes indicó que lo que más desperdicia son los vegetales, un $34 \%$ indicó que lo que más se desperdicia son carbohidratos, $19 \%$ comida chatarra y el $7 \%$ proteínas. 


\section{Gráfico 5}

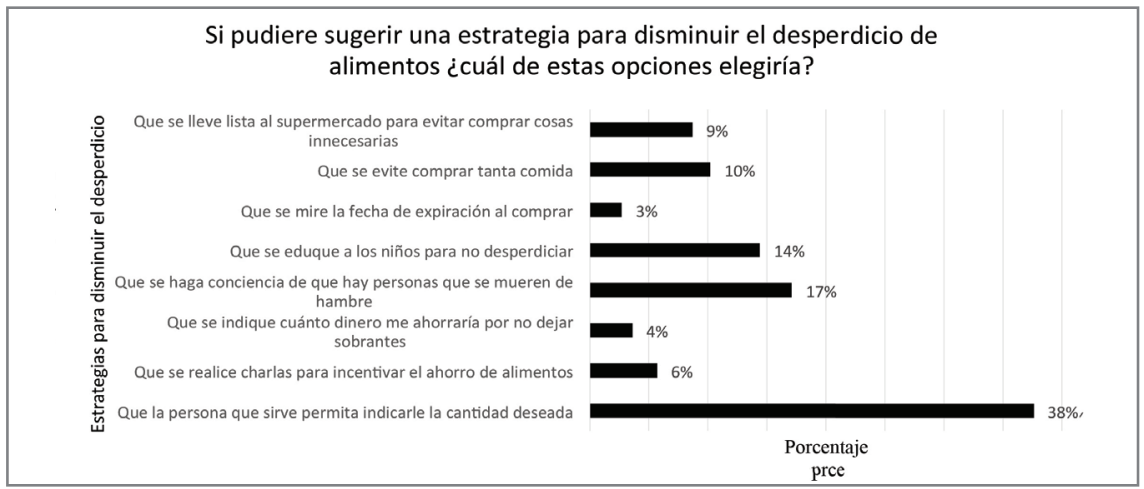

Fuente: Elaboración propia

El gráfico 5 muestra que del 38\% de encuestados lo que más le gustaría es que la persona que sirve le permita indicarle la cantidad de comida deseada, el 17\% sugiere que se haga conciencia que hay personas que se mueren de hambre, el $14 \%$ que se eduque a los niños para que no desperdicien los alimentos, el $10 \%$ que se evite comprar tanta comida, el 9\% que se lleve una lista al supermercado para evitar comprar alimentos que no se necesitan, el 6\% que se realice charlas para incentivar el ahorro de alimentos, que se indique cuanto se ahorra al no desperdiciar alimentos y el 3\% indicó que se mire la fecha de expiración al comprar.

\section{Gráfico 6}

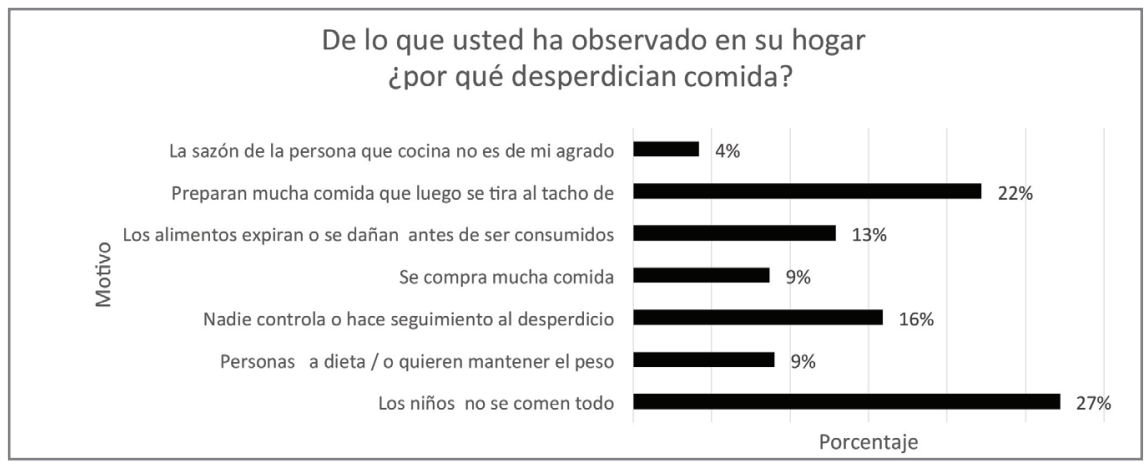

Fuente: Elaboración propia

Como indica el Gráfico 6, el 27\% de encuestados indicó que los niños no se comen todo su alimento, el 22\% indicó que se prepara mucha comida 
que luego termina en el tacho de basura, el 16\% afirmó que nadie controla o hace seguimiento al desperdicio, un $13 \%$ que los alimentos expiran o se dañan antes de ser consumidos, el 9\% las personas que hacen dieta o que compran mucha comida y un $4 \%$ dijo que la sazón no es de su agrado.

\section{Gráfico 7}

\section{¿Ha recibido alguna vez alguna instrucción para no dejar comida?}

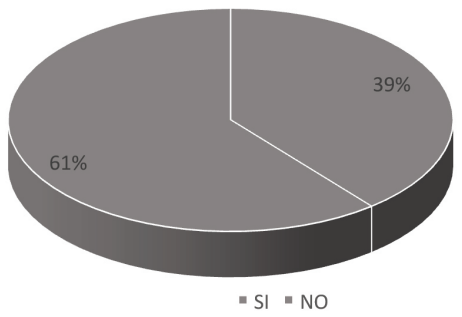

Fuente: Elaboración propia

Del total de encuestados sólo el no ha recibido instrucción alguna lo $39 \%$ indicó haber recibido instruc- cual se observa en el Gráfico 7. ción para no dejar comida y un $61 \%$

\section{Gráfico 8}

\section{¿De parte de quien recibió instrucciones para no dejar comida?}

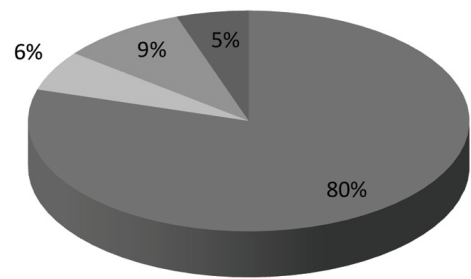

- Padres Docentes Conciencia propia Medios de comunciación, radio, tv, redes sociales, etc. 
El Gráfico 8 muestra que de los 131 estudiantes que si recibieron instrucción para no dejar comida sobrante, el $80 \%$ indicó que fue ins- truido por sus padres, el $9 \%$ por conciencia propia, el $6 \%$ de sus docentes y el $5 \%$ a través de los medios de comunicación.

\section{Gráfico 9}

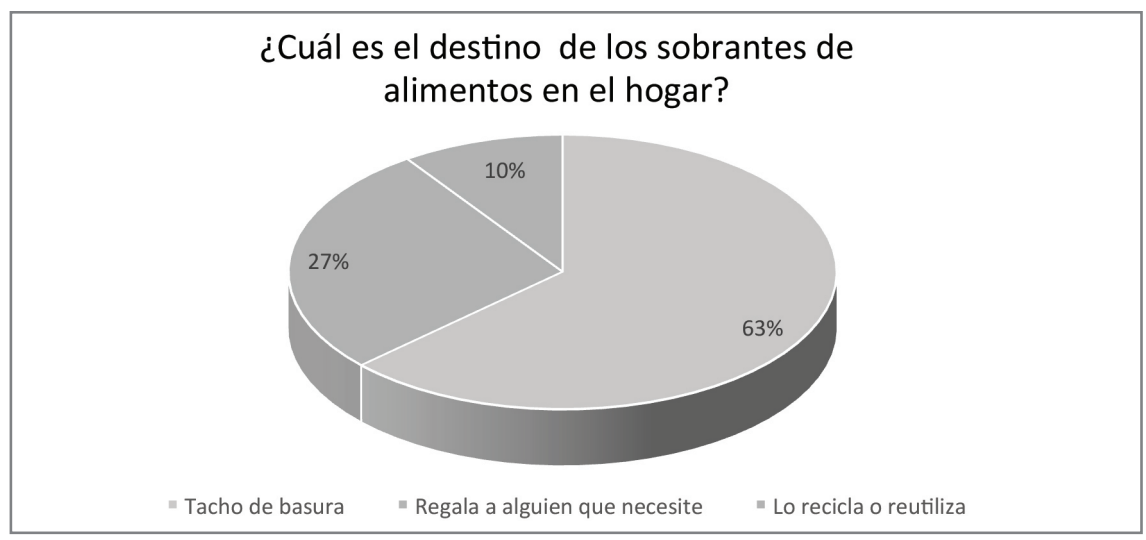

Fuente: Elaboración propia

El Gráfico 9 muestra que un en el tacho de basura, un 27\% afirmó $63 \%$ de encuestados indicó que los que regala a alguien que necesite y un desperdicios de alimentos terminan $10 \%$ lo recicla o reutiliza.

\section{Gráfico 10}

\section{¿Conoce usted el impacto ambiental que produce el desperdicio de alimentos?}

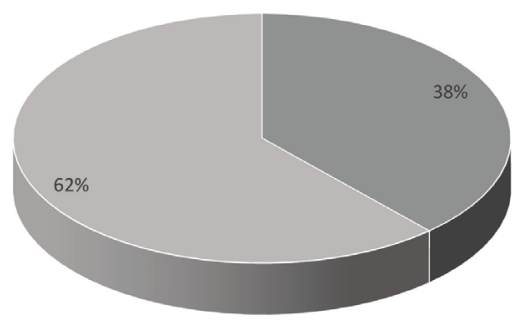

$=\mathrm{SI}=\mathrm{NO}$ 
Al preguntar a los jóvenes si conocen el impacto ambiental del desperdicio de alimentos, un 38\% indicó que si y un $62 \%$ indicó que no, como se observa en el Gráfico 10.

\section{Discusión}

El 32\% de la muestra indica que los jóvenes dejan comida porque no les gusta algún ingrediente de la comida y $31 \%$ porque le sirven mucha comida, lo cual evidencia la costumbre en el Ecuador especialmente en la costa de servir gran cantidad de arroz y porciones que las personas no se van a servir pues, muchas veces tienen las porciones inventariadas y con un precio fijo para el plato, especialmente si son franquicias o cadenas de tiendas de comida rápida, además existe la limitación en algunos sitios de expendio de comidas que no dan la oportunidad de quitar ingredientes que no les gustan al consumidor, como por ejemplo, la cebolla o algún aderezo en particular.

Los jóvenes dejan más comida en restaurantes cuando comen en la calle, especialmente en el almuerzo dado que comen en los lugares cercanos al campus universitario o por su lugar de trabajo, esto podría explicarse porque los ecuatorianos aún valoran mucho comer en sus hogares y además porque el sector de los restaurantes aún no maneja estándares de calidad especialmente en los pequeños negocios de comida, es común en
Ecuador acudir a un restaurante probar la comida y al no ser del agrado del consumidor, éste abandona el plato y no reclama ni recibe devolución de su dinero y tampoco el proveedor se preocupa de preguntar la razón del abandono de la comida.

También se ha podido conocer dado que el cuestionario evidencia los hábitos de alimentación de los ecuatorianos con una dieta basada en carbohidratos, la muestra indicó que el grupo de alimentos que más se desperdicia son los vegetales. Entre los productos con una tasa más alta de desperdicios anuales en el mundo están las frutas y hortalizas, raíces y tubérculos, seguidas por los cereales y el pescado, y en último lugar se ubican los cultivos oleaginosos, carne y productos lácteos (Pérez, 2013).

Además se pudo conocer que los jóvenes que alguna vez recibieron instrucción para no desperdiciar comida lo hicieron por parte de sus padres y sólo un $6 \%$ por parte de los docentes, lo cual permite conocer que los docentes tienen una gran oportunidad y responsabilidad social de abordar este tema en la cátedra, pues los temas ambientales son de interés general. La interacción entre el individuo, la escuela y la comunidad es necesaria para mejorar las condiciones ambientales (Guevara, 2013).

[...] Trabajando con los valores y capacidades del individuo, que es el primer embrión de nuestras so- 
ciedades, la educación puede favorecer el desarrollo sostenible en su dimensión personal y grupal, local y global... (Novo, 2007, p. 415).

Sería idóneo que la Universidad se apoye en las redes sociales para llevar a los jóvenes campañas para reducir el desperdicio de alimentos y de pronto incluirlo en el (Rugel, Nicolalde \& Alava, 2015) Marketing Ecológico que busca aspectos relevantes como concienciación ecológica, ventaja competitiva y posicionamiento de responsabilidad social.

Otra realidad que se confirmó con la encuesta es que el $63 \%$ de los residuos de alimentos terminan en el tacho de basura y que lamentablemente en la ciudad de Guayaquil no se hace recolección de desechos clasificada, por tanto se evidencia un desaprovechamiento de recursos naturales.

Finalmente los resultados indican que el $62 \%$ de los encuestados no conoce el impacto ambiental que produce el desperdicio de alimentos lo cual explica la indiferencia en sus prácticas cotidianas al momento de desechar un alimento.

\section{Conclusiones}

La docencia permite momentos de diálogo con los estudiantes desde varias perspectivas, por lo que es la oportunidad del docente para abordar temas que fomenten, el ahorro de recursos en los jóvenes.
Es necesario fomentar y específicamente impulsar un cambio en la expectativa del consumidor frente a las frutas y hortalizas para que aprenda a aprovechar al máximo estos alimentos teniendo en cuenta sus características nutricionales y restando importancia a la estética, que no siempre es necesaria a la hora de preparar un plato nutritivo y sabroso (Basso, 2013, p. 12).

Difundiendo la necesidad de comprar sólo la comida necesaria, se podría lograr una generalización de hábitos de ahorro en los estudiantes y es probable que del conocimiento se generen propuestas que luego se eleven a estrategias de estado como la "Estrategia más alimento, menos desperdicio" vigente en España desde el año 2013 (Ministerio de Agricultura, Alimentación y Medio Ambiente, 2013).

Se necesita levantar información que permita conocer la situación del desperdicio de alimentos en todas las fases desde la producción, comercialización, transporte y consumo en el Ecuador.

Existe la urgencia de hacer campañas informativas en las aulas de clase, redes sociales y medios de comunicación de la UPS que permitan al estudiante conocer el impacto negativo de desperdiciar alimentos sobre el medio ambiente.

Normalmente en Ecuador las personas que despachan la comida 
no dan al consumidor instrucciones cuando estos requieren de una porción menor de alimentos a la porción normal, o instrucciones de eliminar algún ingrediente que no es del agrado del cliente.

\section{Bibliografía}

Abate, J. (2013). Comida que se bota: el escándalo de los alimentos que terminan en la basura. Tesis Doctoral, Chile. Obtenido de http://repositorio.uchile.cl/ bitstream/handle/2250/116587/ MEMOR I A \% 20 F I N A L F I N A L \% $20 \% 281 \% 29$. pdf?sequence $=1 \&$ is Allowed $=y$

Asamblea Nacional (2008). Constitución de la República del Ecuador. Obtenido de http://www.asambleanacional. gov.ec/documentos/constitucion_ de_bolsillo.pdf

Basso, N. (2013). Las huellas del despilfarro. Alimentos argentinos. Equipo de nutrición y Educación alimentaria , 8-13.

Calixto, R. (2015). Propuesta en Educación Ambiental para la enseñanza del cambio climático. Revista Electrónica Diálogos Educativos, 15(29), 5468. Recuperado el 13 de 09 de 2015, de file://C:/Users/Usuario\%20Pc/ Downloads/Dialnet-PropuestaEnEducacionAmbientalParaLaEnsenanzaDelCam-5159509\%20(1). pdf

CSA (2014). Las pérdidas y el desperdicio de alimentos en el contexto de sistemas alimentarios sostenibles.

Escobar, M. (2012). Comportamiento sustentable y educación ambiental: una visión desde las prácticas culturales. Revista Latinoamericana de
Psicología, 181-196. Obtenido de http://www.scielo.org.co/pdf/rlps/ v44n1/v44n1a17.pdf

FAO (2014a). Iniciativa mundial sobre la reducción de la pérdida y el desperdicio de alimentos.

(2014b). Pérdidas y desperdicio de alimentos en América Latina y El Caribe.

Guevara, J. (2013). Ecología Humana y acción proambiental: alteridades recíprocas aula-escuela-comunidad para el manejo sustentable de residuos. Revista Latinoamericana de Psicología, 447-457. Recuperado el 13 de Septiembre de 2015, de http://www.scielo.org.co/pdf/rlps/ v45n3/v45n3a10.pdf

HLPE (2014). Las pérdidas y el desperdicio de alimentos en el contexto de sistemas alimentarios sostenibles. Un informe del Grupo de alto nivel de expertos en seguridad alimentaria y nutrición del Comité de Seguridad Alimentaria Mundial. Roma, 2014. Obtenido de http://www.fao. org/3/a-i3901s.pdf

INEC (2011-2012). Encuesta Nacional de Ingresos y Gastos de los hogares urbanos y rurales.

Ministerio de Agricultura, Alimentación y Medio Ambiente (2013). Estrategia: Más alimento, menos desperdicio. Madrid: Edita.

Novo, M. (2007). El desarrollo sostenible. Su dimensión ambiental y educativa. Madrid: Pearson Educación S.A.

Pérez, L. (2013). El rol de los consumidores. Reducir el desperdicio para alimentar al mundo. Obtenido de http://www.redalyc.org/comocitar. oa? $\mathrm{id}=86429347004$

Rugel, D., Nicolalde, M., \& Alava, C. (2015). Plan de Marketing ecológico para mitigar los impactos ambientales en la Universidad Po- 
litécnica Salesiana. Retos, 55-72. Recuperado el 13 de 09 de 2015

SENPLADES (2013). Plan Nacional para el Buen Vivir 2013-2017. Obtenido de http://documentos.senplades.gob.ec/Plan\%20Nacional\%20 Buen\%20Vivir\%202013-2017.pdf Xavier, M., \& Gascón, J. (2014). Alimentos desperdiciados. Un análisis del de- rroche alimentario desde la soberanía alimentaria. Barcelona: Icaria Editorial. Recuperado el 06 de 06 de 2016, de http://www.clacso.org.ar/ libreria-latinoamericana-cm/buscar_libro_resultado.php?campo $=$ ti tulo\& texto $=$ desperdicio $+\&$ imageF ield. $x=0$ \& imageField. $y=0$

Recepción: 27-01-2016 - Aceptación: 21-06- 2016 